This item was submitted to Loughborough's Research Repository by the author.

Items in Figshare are protected by copyright, with all rights reserved, unless otherwise indicated.

\title{
Accurate characterisation of hole geometries by fringe projection profilometry
}

PLEASE CITE THE PUBLISHED VERSION

http://dx.doi.org/10.1117/12.2270210

PUBLISHER

(c) SPIE

VERSION

VoR (Version of Record)

LICENCE

CC BY-NC-ND 4.0

REPOSITORY RECORD

Wu, Yuxiang, Harshana G. Dantanarayana, Huimin Yue, and Jonathan M. Huntley. 2019. "Accurate Characterisation of Hole Geometries by Fringe Projection Profilometry". figshare.

https://hdl.handle.net/2134/25980. 


\title{
Accurate characterisation of hole geometries by fringe projection profilometry
}

\author{
Yuxiang $\mathrm{Wu}^{\mathrm{a}}$, Harshana G. Dantanarayana*b ${ }^{*}$ Huimin Yue ${ }^{\mathrm{a}}$, Jonathan M. Huntley ${ }^{\mathrm{b}}$ \\ ${ }^{a}$ University of Electronic Science and Technology of China, Chengdu City, Sichuan Province, \\ China; ${ }^{b}$ Wolfson School of Mechanical, Electrical and Manufacturing Engineering, Loughborough \\ University, Loughborough, Leicestershire, LE11 3TU, UK.
}

\begin{abstract}
Accurate localisation and characterisation of holes is often required in the field of automated assembly and quality control. Compared to time consuming coordinate measuring machines (CMM), fringe-projection-based 3D scanners offer an attractive alternative as a fast, non-contact measurement technique that provides a dense 3D point cloud of a large sample in a few seconds. However, as we show in this paper, measurement artefacts occur at such hole edges, which can introduce errors in the estimated hole diameter by well over $0.25 \mathrm{~mm}$, even though the estimated hole centre locations are largely unaffected. A compensation technique to suppress these measurement artefacts has been developed, by modelling the artefact using data extrapolated from neighboring pixels. By further incorporating a sub-pixel edge detection technique, we have been able to reduce the root mean square (RMS) diameter errors by up to 9.3 times using the proposed combined method.
\end{abstract}

Keywords: fringe projection, measurement artefacts, hole detection, hole diameter estimation, error compensation

\section{INTRODUCTION}

Locating holes and measuring hole geometries in machined parts are often required in quality control and automated assembly ${ }^{1,2}$. The current industry standard method involves the use of coordinate measuring machines (CMM) . $^{3}$ However these require mechanical contact to be made between a probe and the inside of the hole at a number of points, which is thus a time-consuming and costly process. As an alternative, fringe projection profilometry (FPP) techniques can be used to generate a dense 3D point cloud of the measured part(s) in typically just a few seconds. These dense point clouds can include several hundred coordinates in and around a given hole, and allow one to characterize a large number of holes in a single measurement ${ }^{4}$.

In this paper, we (i) investigate experimentally the accuracy of hole geometry characterisation by FPP, (ii) introduce an algorithm to correct for the systematic measurement errors by FPP, and (iii) demonstrate an improvement in hole diameter measurement by up to one order of magnitude through the use of the correction algorithm.

\section{METHOD}

In order to compare hole characterisations by both techniques, a test sample was fabricated out of aluminum as shown in Figure 1. The flat aluminum plate consisted of arrays of circular holes ranging from $30 \mathrm{~mm}$ diameter down to $1 \mathrm{~mm}$ diameter. In total, 90 holes were measured with a mechanical CMM (LK Metris Ultra) by touching the holes along the perimeter at multiple points just below the surface. The CMM probe touched 46 points along the perimeter for the smallest holes, with an increasing number of touch points with increasing diameter, up to 909 points for the largest holes. The measurement process took more than 2 hours even with prior knowledge of the nominal hole locations.

The same plate was measured by a Phase Vision Quartz 1200 FPP scanner. Using Canny edge detection, edge cluster grouping and ellipse fitting to the edge clusters on the texture image, the holes were automatically identified. A circle was subsequently fitted to the $\mathrm{X}, \mathrm{Y}, \mathrm{Z}$ coordinates of the perimeter pixels of each detected ellipse. In this way, all holes from a single scan were automatically identified and characterised, except for the smallest (1 mm diameter) holes, which were blurred and hence could not be reliably detected by the Canny edge detector.

*h.g.dantanarayana@lboro.ac.uk; phone +44 (0)1509 227565; 


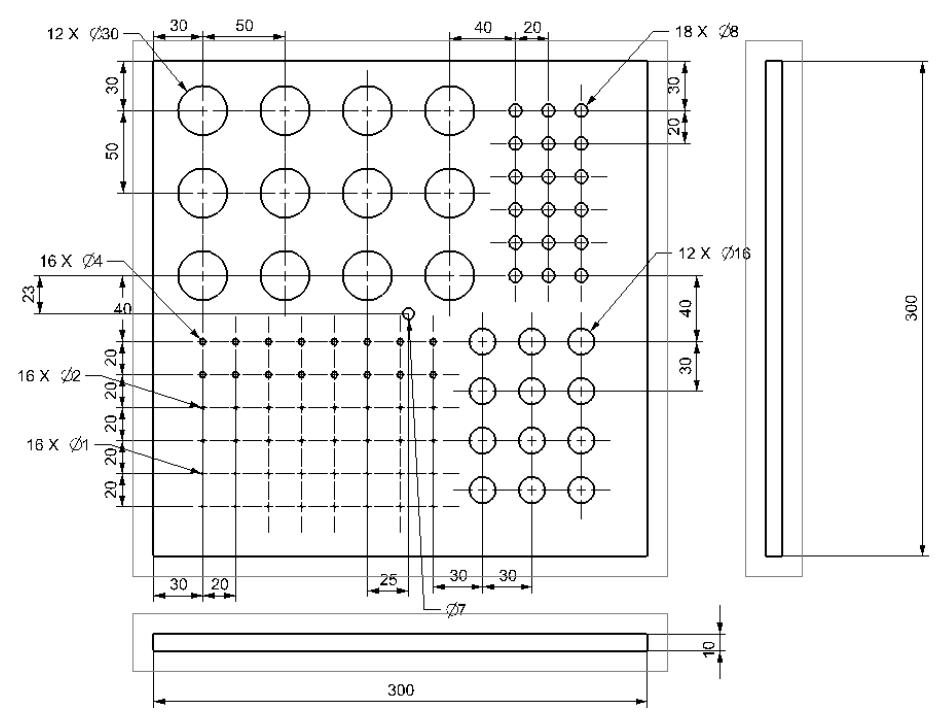

Figure 1. The test panel with arrays of holes, the diameters of which range from $30 \mathrm{~mm}$ down to $1 \mathrm{~mm}$.

Figure 2 shows the estimated radii of a set of $16-\mathrm{mm}$ diameter holes of the test panel. All radii were overestimated by values in the range $0.24 \mathrm{~mm}-0.32 \mathrm{~mm}$. In comparison, CMM measurements reported only up to $0.025 \mathrm{~mm}$ deviation from the nominal values. For all holes between $2 \mathrm{~mm}-30 \mathrm{~mm}$ diameter, the root mean square (RMS) error $(0.22 \mathrm{~mm}$ $0.47 \mathrm{~mm}$ for hole diameters in the range $30 \mathrm{~mm}$ down to $2 \mathrm{~mm}$ ) was $9-19$ times larger than the measurement uncertainty of the CMM.
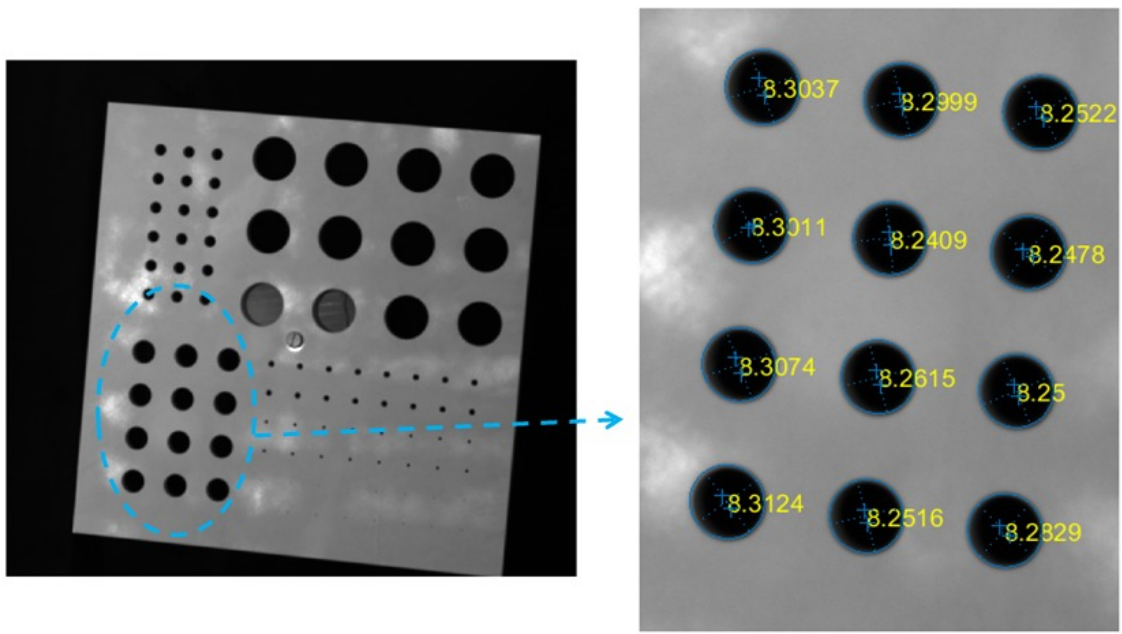

Figure 2. Radii estimation results (units: $\mathrm{mm}$ ) obtained from the ellipse fitting to the $3 \mathrm{D} \mathrm{X}, \mathrm{Y}, \mathrm{Z}$ data on the edge pixels, measured with the fringe projection 3D scanner.

The discrepancy in estimated hole radius by FPP measurements can be explained by the measurement artefacts occurring around the edges of the holes, as shown in Figure 3(a) for an $8 \mathrm{~mm}$ diameter hole. Biasing of the measured height values 
by up to $0.5 \mathrm{~mm}$ is seen to occur at the hole edges. As a result, hole radii are always overestimated due to the effect illustrated in Figure 3(b).

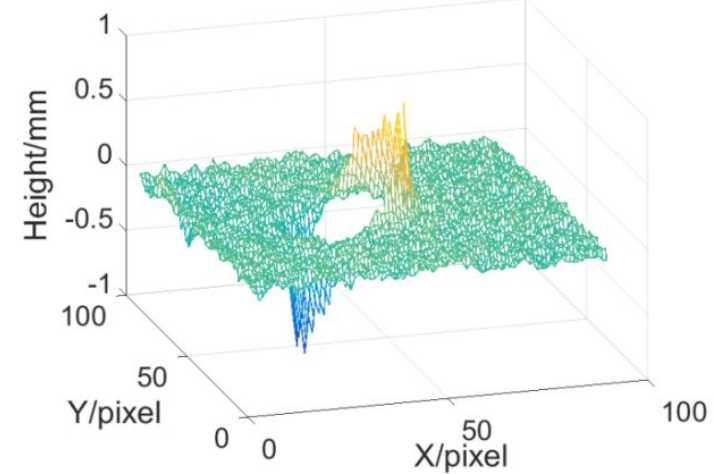

(a)

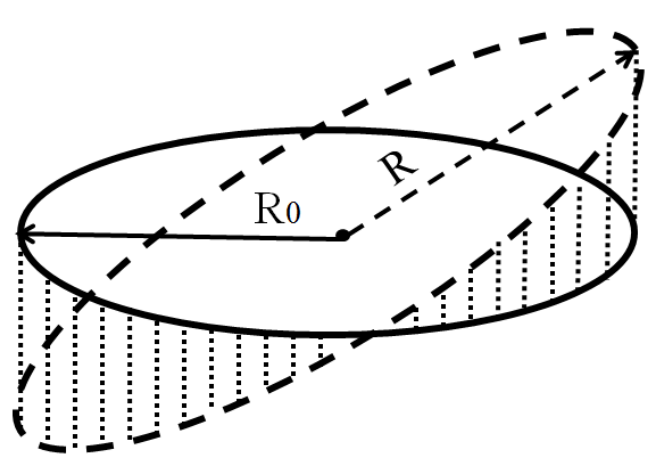

(b)

Figure 3. Measurement artefacts around the edge of a hole. (a) Experimental height distribution of an $8 \mathrm{~mm}$ diameter hole measured by fringe projection; (b) an illustration of how these measurement artefacts affect the diameter estimation.

These artefacts are believed to occur as a result of the finite size of the point spread function (PSF) of the camera within the FPP system. The size of the PSF region on the object, from which light is received by a given point in the image plane of the camera, is at a minimum the pixel footprint, i.e. the size of the pixel divided by the camera magnification. In practice, it is often much larger due to lens aberrations or camera defocus. The intensity and unwrapped phase measured at each pixel are averages of the intensity and unwrapped phase values over the pixel footprint ${ }^{5}$. In the absence of discontinuities, the average values of intensity and unwrapped phase values are close to the values at the centre of the pixel footprint of the measured object. However, at a discontinuity or absence of a surface (such as a hole), part of the signal is missing. Depending on whether the missing portion is from the region with higher than average fringe phase, or lower than average fringe phase, the measured phase is biased in the downward and upward directions, respectively. This is believed to be the cause of the negative and positive artefacts on the two sides of the hole shown in Figure 3(a). Holes contain both intensity and geometrical discontinuities and these pixels cannot be simply eliminated from the measurements as the edge points are the most critical in characterising hole geometries.

\section{THEORY}

In order to estimate these measurement artefacts and eventually compensate for them, we identify two pixel regions in a local neighborhood of a hole edge discontinuity. Pixels within the radius, $R_{P}$, of the PSF from the edge are the directly affected pixels that need to be compensated. The pixels just outside this PSF region, within a band of width equal to the PSF radius, are unaffected by the phenomenon described in the previous section, and so can be used to compensate the data in the affected pixels. Here we assume that the test object has the same surface scattering characteristics over a region up to a distance $2 R_{P}$ away from a discontinuity in a direction normal to the edge.

The Phase Vision scanner projects both a vertical and a horizontal fringe sequence. The measured unwrapped phase maps for the two sequences are denoted $\widetilde{\omega}_{x}(x, y)$ and $\widetilde{\omega}_{y}(x, y)$, with corresponding intensity images denoted $\tilde{I}_{x}(x, y)$ and $\tilde{I}_{y}(x, y)$, respectively. Note that $(x, y)$ represents coordinates in the pixel domain of the image. We first approximate the 'true' unwrapped phases and intensities within the PSF zone by $\widehat{\omega}_{x}(x, y)$ and intensity $\hat{I}_{x}(x, y)$, which are calculated as a first degree and zeroth degree polynomial respectively from the nearby portion of the 'unaffected zone' outside the PSF region. These two functions effectively extrapolate the unwrapped phase and intensity from the unaffected region into the affected region. For a given affected pixel $\left(x_{c}, y_{c}\right)$, the pixel band outside the PSF that is used to calculate $\widehat{\omega}_{x}$ is selected as an annular section of length $2 R_{P}$ and width $R_{P}$, as illustrated by the shaded patch in Figure 4 below.

The unwrapped phase $\bar{\omega}_{x}\left(x_{c}, y_{c}\right)$ that we expect to see in the affected region as a result of the edge discontinuity is modelled as a weighted average, over the PSF, of the $\widehat{\omega}_{x}$ values at the pixels surrounding $\left(x_{c}, y_{c}\right)$. We have assumed that 
the modelled unwrapped phase is weighted by the intensity and that the PSF has the shape of a Gaussian, where $R_{P}$ is the full width half maximum value.

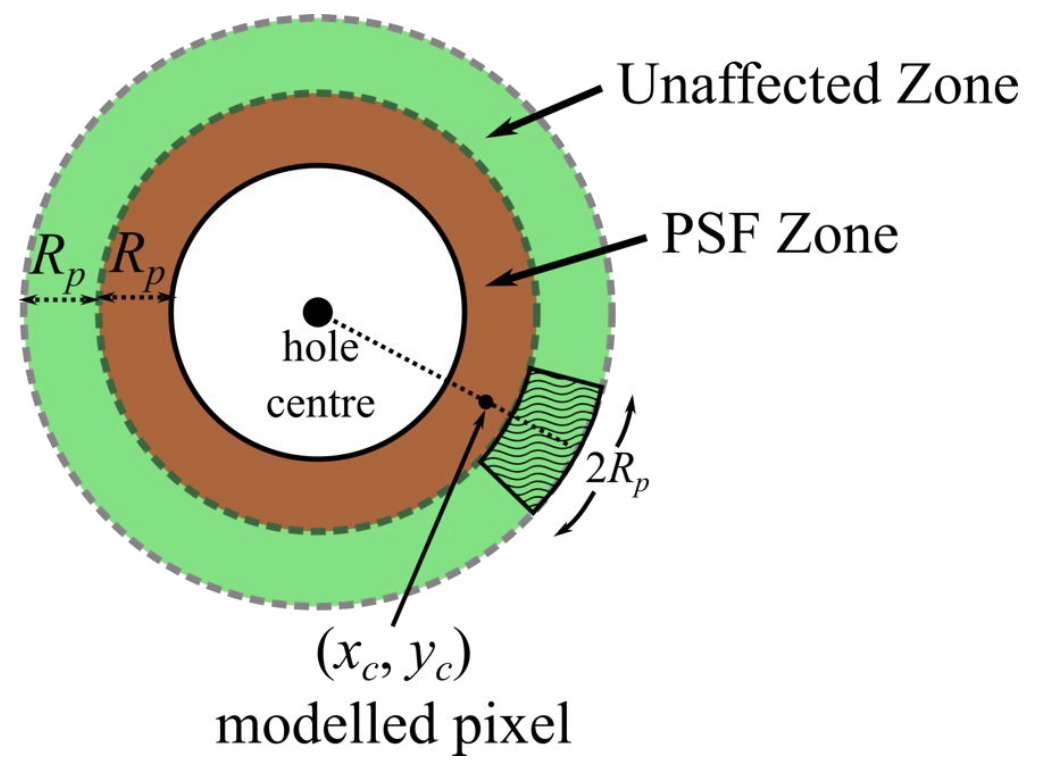

Figure 4: schematic of the PSF zone and the surrounding unaffected zone around a hole (solid line). The shaded patch denotes the area over which $\widehat{\omega}_{x}$ and $\widehat{\omega}_{y}$ are calculated for pixel $\left(x_{c}, y_{c}\right)$.

The modelled unwrapped phase of a point $\left(x_{c}, y_{c}\right)$ is then given by the convolution integral

$$
\bar{\omega}_{x}\left(x_{c}, y_{c}\right)=\frac{\int_{-\infty}^{\infty} \int_{-\infty}^{\infty} \hat{\omega}_{x}(x, y) \hat{I}_{x}(x, y) P S F\left(x-x_{c}, y-y_{c}\right) \mathrm{d} x \mathrm{~d} y}{\int_{-\infty}^{\infty} \int_{-\infty}^{\infty} \hat{I}_{x}(x, y) P S F\left(x-x_{c}, y-y_{c}\right) \mathrm{d} x \mathrm{~d} y},
$$

where the point spread function is defined as

$$
\operatorname{PSF}\left(x-x_{c}, y-y_{c}\right)=e^{-\frac{\left(x-x_{c}\right)^{2}}{2 c^{2}}} e^{-\frac{\left(y-y_{c}\right)^{2}}{2 c^{2}}},
$$

and where $c$ is related to $R_{P}$ through the equation $R_{P}=2 \sqrt{2 \ln 2} c$.

The compensated unwrapped phase $\widehat{\omega}_{x}^{\prime}\left(x_{c}, y_{c}\right)$ within the PSF region is then obtained by subtracting the estimated error due to the edge discontinuity from the measured phase as follows:

$$
\widehat{\omega}_{x}^{\prime}\left(x_{c}, y_{c}\right)=\widehat{\omega}_{x}\left(x_{c}, y_{c}\right)-\left(\left(\bar{\omega}_{x}\left(x_{c}, y_{c}\right)-\widetilde{\omega}_{x}\left(x_{c}, y_{c}\right)\right)\right. \text {. }
$$

\section{RESULTS}

The flat aluminium plate shown in Figure 1 was measured by the Phase Vision Quartz 1200 fringe projection 3D scanner at a distance of $2.5 \mathrm{~m}$, with an image resolution of $2048 \times 2048$ pixels. The compensation algorithm described in the previous section was used to calculate corrected phase maps on and around hole edge pixels that had previously been identified from the intensity image. The scanner employs a reverse exponential sinusoidal fringe sequence and a leastsquares temporal phase unwrapping (TPU) algorithm ${ }^{6,7}$ to calculate the unwrapped phase from the recorded fringe patterns. Phase values are converted to coordinates using a photogrammetric pinhole model for camera and projector, where the camera and projector parameters are determined using a bundle adjustment procedure ${ }^{8}$. The radius of the PSF 
was estimated from a vertical black and white stripe board placed at the same position as the aluminium plate in the above experiment ${ }^{9}$, giving the value $R_{P}=5$ pixels at $2.5 \mathrm{~m}$.

Figure 5 shows the height distribution around a $8 \mathrm{~mm}$ diameter hole before and after the unwrapped phase compensation. The measurement artefact is well suppressed after phase compensation, thus providing the basis for a more accurate estimation of hole diameter.

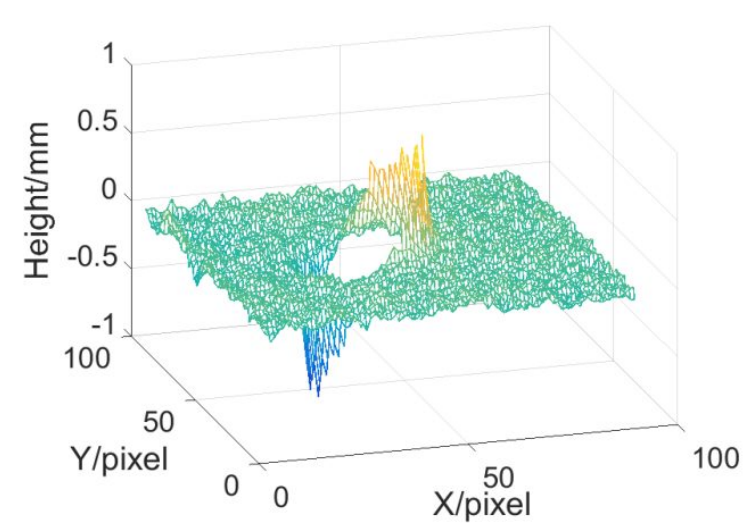

(a)

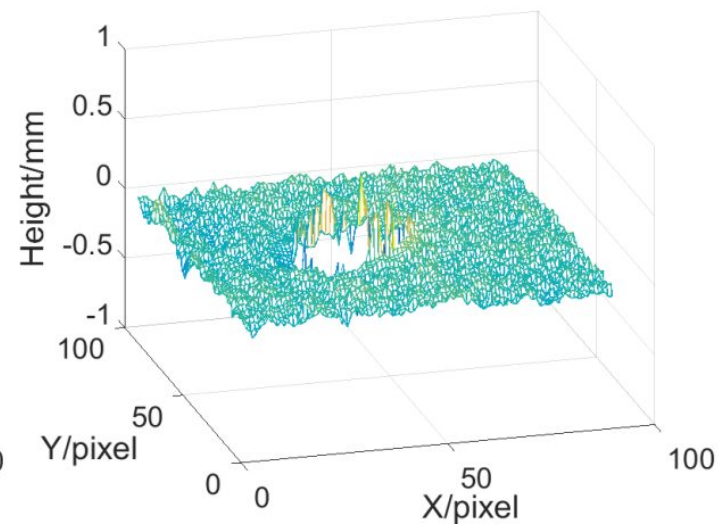

(b)

Figure 5: Measured height distribution around an $8 \mathrm{~mm}$ diameter hole (a) before and (b) after unwrapped phase compensation.

In addition to the suppression of the measurement artefact, accurate detection of the edge pixel locations using the intensity image is essential in order to obtain an accurate estimate of the hole geometry. A sub-pixel detection technique, developed by Trujillo-Pino et. al. ${ }^{10}$, and a Fourier descriptor (FD) method in which the hole edge contour radius is expanded as a Fourier series in azimuthal angle about the hole centre, were employed for this purpose. The required X, $\mathrm{Y}$ and $\mathrm{Z}$ coordinate data were calculated at the sub-pixel hole edge locations from the compensated unwrapped phase values, by linear interpolation between the four nearest pixels. The diameters and centres of all holes were then estimated by least-squares fitting 3D circles to each $\mathrm{X}, \mathrm{Y}, \mathrm{Z}$ sub-pixel hole edge data set. The radii and centre estimations thus obtained from the experiment were then compared with the corresponding measurements obtained by the CMM. The measurement coordinate frame of the FPP scanner was rotated and translated to that of the CMM measurement

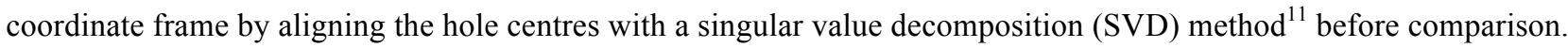

The root mean square (RMS) deviation of the estimated diameter from that given by the CMM measurements was calculated for each hole set when using the FD sub-pixel edge detection method, and plotted in Figure 6 below. In addition, the RMS deviation of the identified hole centres from the CMM hole centres was calculated for each hole set and plotted in the same figure below. Figure 6 confirms that the measurement artefact suppression combined with the sub-pixel detection can sucessfully bring down the error by 3.6-9.3 $\times$ for holes larger than or equal to $4 \mathrm{~mm}$ diameter.

However, for the $2 \mathrm{~mm}$ diameter holes, the diameter estimation result was only improved by $1.4 \times$. The number of pixels across the diameter of a hole is about 10 in the intensity image for $2 \mathrm{~mm}$ diameter holes, when the flat aluminium plate was placed at $2.5 \mathrm{~m}$, which is at the centre of the working envelope of the FPP scanner. Since the PSF radius is 5 pixels, the $2 \mathrm{~mm}$ hole diameter is about equal to $2 R_{P}$ making this the limiting case for the validity of the model given in equation (1). The $1 \mathrm{~mm}$ holes could not be identified by edge detection as their diameter was approximately equal to $R_{P}$. It should be noted that the experimental setup used in this study had a field of view of about $1 \times 1 \mathrm{~m}^{2}$ at a distance of $2.5 \mathrm{~m}$, and holes smaller than $4 \mathrm{~mm}$ diameter could be better estimated with a system that has a narrower field of view and a shorter working distance, so that there are more than $2 R_{P}$ pixels across the diameter.

According to Figure 6, one can see by contrast that the centre estimation is barely altered by the compensation across the full range of hole diameters. This can be explained by the anti-symmetrical nature of the measurement artefact, shown schematically in Figure 3(b): the best fit circle is tilted and stretched, but its centre location remains unchanged. 


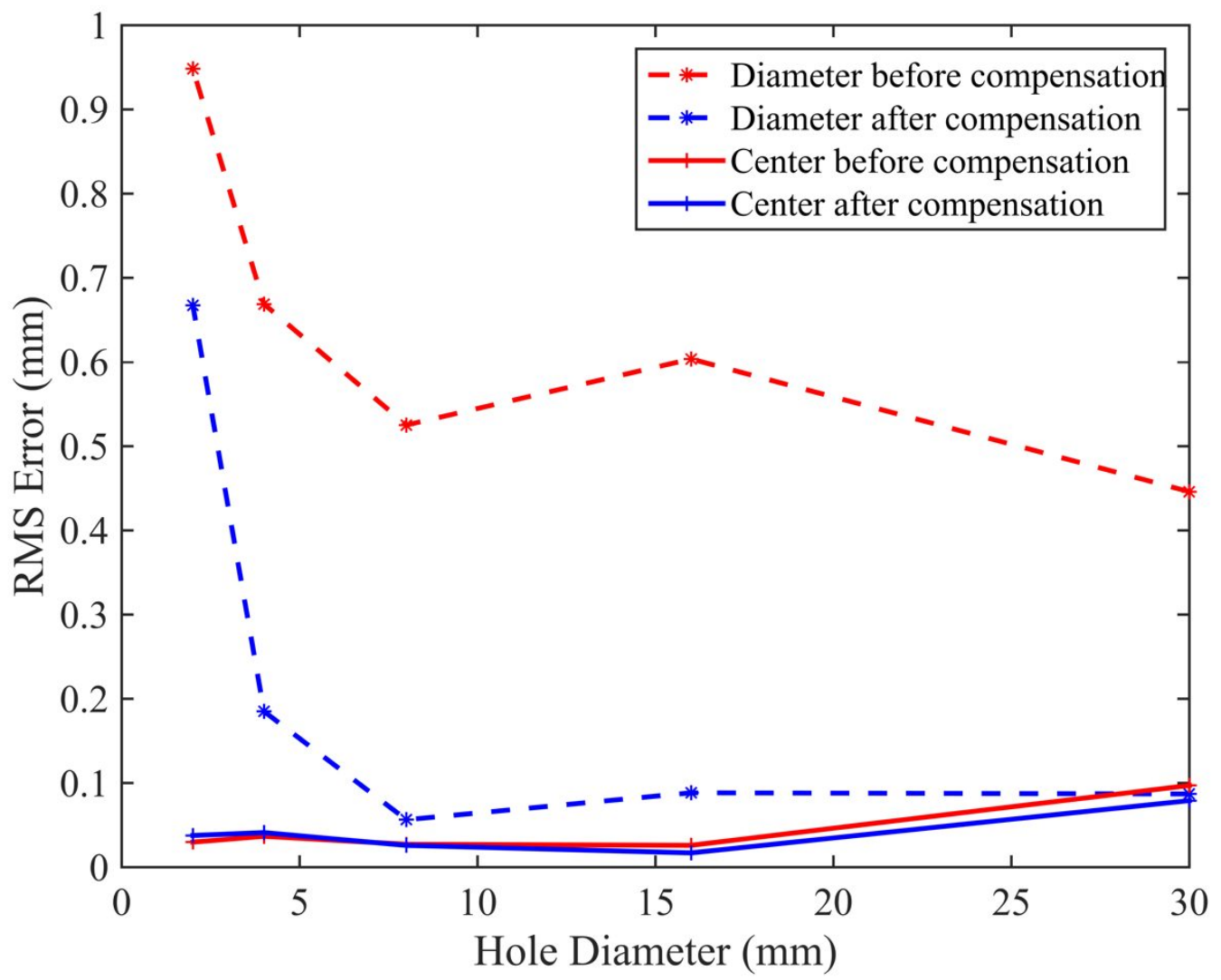

Figure 6. Estimated diameter and centre errors before and after measurement artefact suppression, for holes with diameters in the range $2-30 \mathrm{~mm}$.

\section{CONCLUSIONS}

Automated assembly and part verification often requires the ability to identify and characterise holes in manufactured components, and fringe projection based 3D scanners can provide an attractive alternative to time consuming CMMs, as a non-contact fast measurement technique. However, we have found evidence that measurement artefacts occur at hole edges, in the very region where accurate measurements are required for the estimation of hole geometry. A method has been developed to successfully suppress these measurement artefacts. It relies on modelling and compensating for the errors in the unwrapped phase of the affected regions, i.e., on and around the hole edge pixels, using the extrapolated values from neighboring pixels. By further incorporating a sub-pixel edge detection method, the RMS error of the diameter relative to the CMM measurements has been reduced down to the range $0.08 \mathrm{~mm}-0.66 \mathrm{~mm}$ for hole diameters ranging from $30 \mathrm{~mm}$ to $2 \mathrm{~mm}$, which represents an improvement of $1.4-9.3 \times$ over the uncompensated results.

\section{ACKNOWLEDGMENTS}

The authors are grateful to Ben Horton for the design of the test panel, and acknowledge financial support from the Engineering and Physical Sciences Research Council under the Light Controlled Factory project EP/K018124/1.

\section{REFERENCES}

[1] Munkelt, C., Kühmstedt, P., Aschermann, L. and Seidel, F., "Automatic complete high-precision optical 3D measurement of air cooling-holes of gas turbine vanes for repair," Proc. SPIE 9525, 9525121—95251217, (2015). 
[2] Tian, G.Y., Zhao, Z.X., Baines, R.W. and Corcoran, P., "A miniaturised sensor for deep hole diameter measurement," Precis. Eng. 23, 236-242 (1999).

[3] Flack, D., "Measurement Good Practice Guide No.41 - CMM measurement strategies," Teddington : National Physical Laboratory, (2014).

[4] Gorthi, S.S., and Rastogi, P., "Fringe Projection Techniques: Whither we are?" Opt. Lasers Eng. 48(2), 133140 (2010).

[5] Brakhage, P., Heinze, M., Notni, G. and Kowarschik, R., "Influence of the pixel size of the camera on 3Dmeasurements with fringe projection," Proc. SPIE 5144, 478-483 (2003).

[6] Saldner, H.O. and Huntley, J. M., "Temporal phase unwrapping: application to surface profiling of discontinuous objects," Appl. Opt. 36(13), 2770-2775 (1997).

[7] Huntley, J.M. and Saldner, H.O., "Error-reduction methods for shape measurement by temporal phase unwrapping," J. Opt. Soc. Am. A 14(12), 3188-396 (1997).

[8] Huntley, J.M., Ogundana, T., Burguete, R.L. and Coggrave, C.R., "Large-scale full-field metrology using projected fringes: some challenges and solutions," Proc. SPIE 6616, 66162C (2007).

[9] Wang, F.P., "The Measurement of the Point Spread Function in Optical System with CCD," JGTC Intl. J. of Gannan Teachers College 06, 17-18 (2005).

[10] Trujillo-Pino, A., Krissian, K., Alemán-Flores, M. and Santana-Cedrés, D., “Accurate subpixel edge location based on partial area effect," Image Vis. Comput. 31(1), 72-90 (2013).

[11] Sjödahl, M. and Oreb, B.F., "Stitching interferometric measurement data for inspection of large optical components," Opt. Eng. 41(2), 403-408 (2002). 